\title{
Large Retroperitoneal Desmoid-Type Fibromatosis of Renal Origin: Case Report and Literature Review
}

\author{
Tarun Jindal ${ }^{1}$, Ankush Sarwal ${ }^{2 *}$, Pravin Ramdas Pawar ${ }^{1}$, Piyush Shankar Tripathi ${ }^{2}$ \\ ${ }^{1}$ Department of Uro-oncology. Tata Medical Centre, Kolkata, India \\ ${ }^{2}$ Department of Surgical oncology. Tata Medical Centre, Kolkata, India
}

"Corresponding author: Ankush Sarwal, Department of Surgical oncology. Tata Medical Centre, Kolkata, India. Tel: +919812636877; Email: drankushsarwal@yahoo.in

Citation: Jindal T, Sarwal A, Pawar PR, Tripathi PS (2019) Large Retroperitoneal Desmoid-Type Fibromatosis of Renal Origin: Case Report and Literature Review. J Surg 13: 1231. DOI: 10.29011/2575-9760.001231

Received Date: 10 June, 2019; Accepted Date: 14 June, 2019; Published Date: 18 June, 2019

\begin{abstract}
Desmoid tumours are a rare type of tumours. Retroperitoneal desmoid is an uncommon mesenchymal neoplasm presenting as a firm mass with locally aggressive features. Desmoid tumour account for $0.03 \%$ of all tumours and $3 \%$ of all the soft tissue tumours. It usually manifests as a well-circumscribed or ill-defined, solid mass on cross-sectional imaging. The confirmatory diagnosis of these tumours is made by histological examination and immunohistochemistry. Surgical resection of the tumour is the treatment of choice in Desmoid tumours and also helps in clearing the diagnostic dilemma it creates in patient because of its rare nature. Here, we present a rare case of a Large Retroperitoneal Desmoid-type Fibromatosis of renal origin in a 28-year-old female, which was successfully treated surgically at specialised oncology centre in India.
\end{abstract}

\section{Introduction}

Desmoid-Type Fibromatosis (DF), also called desmoid tumour, is an uncommon mesenchymal neoplasm composed of fibrous soft-tissue proliferation [1]. The tumour is characterized by locally aggressive growth but rarely metastasize. Desmoid tumour may arise from anywhere in the body, but the common site being extra-abdominal $(60 \%)$, abdominal wall $(25 \%)$, and intraabdominal (8-15\%). Retroperitoneal DF is a rare clinical entity and accounts for less than $1 \%$ of retroperitoneal masses [2]. Desmoid tumour account for $0.03 \%$ of all tumours and $3 \%$ of all the soft tissue tumours. The incidence is 2.4-4.3 new cases per million people per annum. It occurs most often in age group between 20 to 35 years of age, with much more incidence in females as compared to males. Surgical resection of the tumour is the treatment of choice in DF and also helps in clearing the diagnostic dilemma it creates in patient because of its rare nature [3]. Here, we present a rare case of a Large Retroperitoneal Desmoid-type Fibromatosis of renal origin in a 28-year-old female, which was successfully treated surgically at specialised oncology centre in India.

\section{Case Report}

A 28-year-old female patient with a BMI of $21.35 \mathrm{~kg} /$ $\mathrm{m}^{2}$, without significant surgical history or previous comorbidity presented to the outpatient clinic department with symptoms of nausea and vomiting for last 8 months, for which she has been taking symptomatic treatment form local practitioner for last 6 months. For last 2 months she has developed a lump in abdomen for which she had an ultrasonography of abdomen, which showed a $161 \times 155 \mathrm{~mm}$ mass arising from the upper pole of left kidney. She could not recall any hospitalization or abdominal trauma in the past. On physical examination, there was hard lump palpable in the abdomen of size around $20 \times 15 \mathrm{~cm}$ in the left lumbar region extending into Umbilical, Left hyochodrium and epigastric region. A CT scan (Figure 1) was done which showed a large lobulated heterogenous hypodense retroperitoneal lesion with enhancement within the lesion without calcification. 


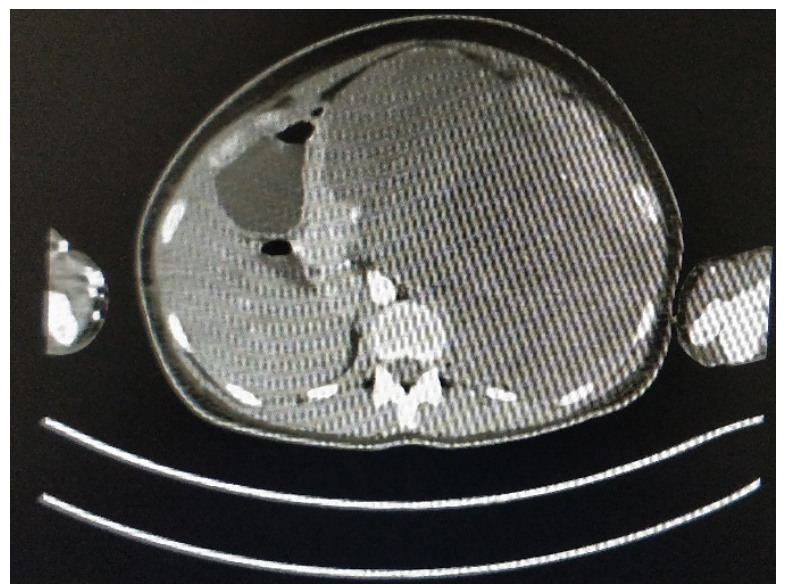

The lesion measured $21.9 \times 18.9$ x $10.3 \mathrm{cms}$. The Lesion was not separate from left kidney and suprarenal region and was displacing spleen anteriorly and towards left. Stomach was displaced to the right and pancreas was displaced anterior and surrounded by lesion. Left Ureter other retroperitoneal organs were normal without significant retroperitoneal Lymphadenopathy. CT Thorax which showed no thoracic metastasis. Ultrasound Guided Biopsy was done from the growth which favoured the growth to be Desmoid tumour. Based on the symptoms as well as radiological and histological correlation, patient was planned for Explorative laparotomy. Intraoperatively, there was a large retroperitoneal mass of size around $20 \times 20 \mathrm{~cm}$ was seen occupying the whole left abdomen. Mass was seen infiltration the left kidney and spleen. The mass was densely adherent to Distal pancreas and greater curvature of stomach. Based on these findings En-bloc removal of mass along with Left nephrectomy, Splenectomy, Distal pancreatectomy and resection of wall of greater curvature was done (Figure 2). Primary repair of pancreas and stomach was done. One drain in sub hepatic space and one drain in pelvis was inserted and abdomen was closed in layers.

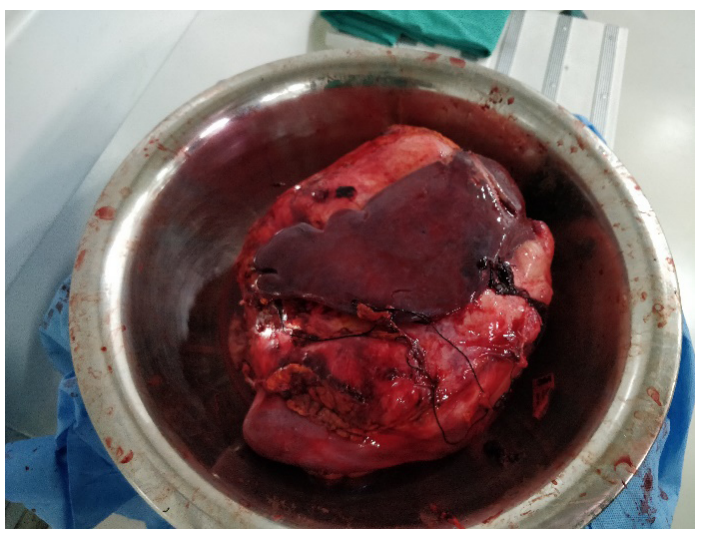

Histopathological examination of the specimen showed tumour of size $22 \mathrm{~cm}$ in greatest dimensions. Tumour is seen infiltrating the renal pelvis and abutting spleen, distal pancreas and gastric wall. All the margins were free of tumour. Histological sections showed a tumour composed of spindle cells, showing mild to moderate atypia, arranged in fascicles with interspersed small arterioles. Mitotic count less than 1/10 high power field. Immunohistochemistry showed tumour cells to be positive for B-catenin, SMA and desmin, while negative for S100 and DOG1, suggesting it to be desmoid-type fibromatosis.

Postoperatively, Patient was shifted to intensive care unit for post-operative management for 3 days and kept nil by mouth for 3 days with intravenous parental nutrition and albumin. Consequently, the patient was gradually shifted to oral liquid diet then soft diet. Sub hepatic drain and pelvis output was reported initially high and was clear in colour. Gradually, the drain output reduced in amount to less than $50 \mathrm{cc}$ per day. Sub hepatic drain was removed on $5^{\text {th }}$ post-operative day and Pelvis drain was removed on $7^{\text {th }}$ post-operative day. The patient recovered well and was discharged on the $8^{\text {th }}$ post-operative day. Post-operatively in view of R0 resection and no distant metastasis, no further adjuvant therapy was given to the patient. The patient has been asymptomatic for 12 months, on clinical and radiological evaluation she is recurrence free and living a healthy normal life style.

\section{Discussion}

Desmoid-Type Fibromatosis (DF) also called desmoid tumour or musculoaponeurotic fibromatosis, is an uncommon mesenchymal neoplasm composed of fibrous soft-tissue proliferation with the production of intracellular collagen [1]. The disease was first described by Mueller in 1838 as a band, or a cyclic structure, and the term fibromatosis as a group of similar changes, was first used by Stout in 1961 [1,4]. Desmoid tumour may arise from anywhere in the body, but the common site being extra-abdominal $(60 \%)$, abdominal wall $(25 \%)$, and intra-abdominal (8-15\%). Retroperitoneal DF is a rare clinical entity and accounts for less than $1 \%$ of retroperitoneal masses [2]. Desmoid tumour account for $0.03 \%$ of all tumours in literature and $3 \%$ of all the soft tissue tumours. The incidence is 2.4-4.3 new cases per million people per annum. The incidence of DF is common in young females between $3^{\text {rd }}$ and $4^{\text {th }}$ decade of life. DF has been associated with multiple risk factors including previous surgery, genetic mutations of the adenomatous polyposis coli gene such as in FAP or the beta-catenin gene, trauma, pregnancy, and oral contraceptive use However, the pathogenesis of DF is not completely understood [3]. The patient can be asymptomatic or could present with vague abdominal symptoms and abdominal lump. On Radiological imaging DF appears as well-defined solid mass of variable attenuation and enhancement, which depends on tissue components. DF with a highly collagenous stroma usually displays homogeneous, soft-tissue attenuation on CT scans. The soft-tissue component can often mimic similar to other solid tumours, such as gastrointestinal stromal tumor, lymphoma, or 
soft tissue sarcoma [5]. Imaging exams are useful in establishing size, extension and anatomical relationship, the confirmatory diagnosis of these tumours is made by histological examination and immunohistochemistry. The cells usually have a poorly circumscribed pattern with spindle cell proliferation forming long beams or spiral patterns and are strongly positive to vimentin staining and the immunoreactivity to beta-catenin is expressed in the $67-80 \%$ of cases. Immunohistochemically, the tumour cells are negative for CD34, CD117, and S-100 protein [6] The main therapeutic strategy is to prevent the invasion of surrounding tissues. The therapeutic decision requires the approach of a multidisciplinary team. Surgery is considered the treatment of choice whenever possible, other alternatives include radiotherapy, hormone therapy, treatment with NSAIDs and even observation have also been reported. In a study done by cates et al, a positive or close $(<1 \mathrm{~mm})$ resection margin was an independent prognostic factor for local recurrence in a multivariate Cox regression model accounting for patient age and anatomic site (hazard ratio 9.52; $\mathrm{P}=0.028$ ), thus emphasizing the need for $\mathrm{R} 0$ resection in such cases [7]. In present case Desmoid-type Fibromatosis had renal origin and which has been rarely reported in literature.

\section{Conclusion}

Desmoid tumours are rare clinical entity. We report a rare case of a Large Retroperitoneal Desmoid-type Fibromatosis of renal origin in a 28 -year-old female, which was successfully treated surgically at specialised oncology centre in India. Surgical resection of the tumour is the treatment of choice in DF and also helps in clearing the diagnostic dilemma it creates in patient because of its rare nature and requires a multidisciplinary team approach.

\section{References}

1. Goldblum JR, Fletcher JA (2013) Desmoid-type fibromatoses. In: Fletcher CDM, Bridge JA, Hogendoorn PCW, Mertens F, editors. WHO classification of tumors of soft tissue and bone. 4th ed. Lyon: IARC Press 2013: 72-73.

2. Destri GL, Ferraro MJ, Calabrini M, Pennisi M, Magro G (2014) Desmoid-type fibromatosis of the mesentery: report of a sporadic case with emphasis on differential diagnostic problems, Case Rep Med 2014.

3. Wang Z, Wu J, Lv A, Tian X, Hao C (2018) En bloc resection for intraabdominal/retroperitoneal desmoid-type fibromatosis with adjacent organ involvement: A case series and literature review. Biosci Trends 12: $620-626$

4. Meazza C, Bisogno G, Gronchi A, Fiore M, Cecchetto G, et al. (2010) Aggressive fibromatosis in children and adolescents: the italian experience. Cancer 116: 233-240.

5. Rajiah P, Sinha R, Cuevas C, Dubinsky TJ, Bush WH Jr, et al. (2011) Imaging of uncommon retroperitoneal masses. Radiographics 31: 949-976.

6. Carlson JW, Fletcher CD (2007) Immunohistochemistry for betacatenin in the differential diagnosis of spindle cell lesions: Analysis of a series and review of the literature. Histopathology 51: 509-514.

7. Cates JM, Stricker TP (2014) Surgical resection margins in desmoidtype fibromatosis: a critical reassessment. Am J Surg Pathol 38: 17071714. 